\title{
Datawing File Sharing and PC Control Application
}

\author{
Mr. Nayan A. Sawant ${ }^{1}$, Ms. Snehal S. Zore ${ }^{1}$, Ms. Dhanashri S. Salunkhe ${ }^{1}$, Mr. Parag S. Joshi ${ }^{1}$ \\ Computer Department, Rajendra Mane College of Engineering and Technology, Ambav, India ${ }^{1}$
}

\begin{abstract}
Users are frustrated with slow transfer speed and carrying cable everywhere with them to transfer file from one device to another so here we introduce DataWing. It will be an application to transfer files from Wi-Fi device to another compatible Wi-Fi device. User can use it to transfer files including photos, videos, music, apps and any other files. It should be supportable for many platforms to transfer file directly by Ad-hoc Wi-Fi connection. In additional DataWing also including services like WebShare and Remote PC Control.
\end{abstract}

Keywords: Android, Java, HTTP, FTP.

\section{INTRODUCTION}

Datawing is an application to transfer files from a Wi-Fi compatible device to an- other similar one. Users can use it to transfer files including photos, videos, music, apps and any other files. It allows Windows, Android devices to transfer files directly by ad-hoc Wi-Fi connections. In addition to transferring files, Datawing services like Web Share [3] and Remote PC Control [1]. The app will offer faster speed than Bluetooth and even Near Field Communication [1], and it is totally secure, considering the type of connection it uses to transfer files. In case of Bluetooth, the speed is adequate, while the USB drive [2] case has a negative side. It is applicable in college and places where connecting USB sticks are not allowed. Remote PC Control [1] is one of the feature of Datawing application. This feature attempt to simplify the task of controlling many different program from one app. Such as YouTube, VLC, Media player etc. This application proposes many remote control for various programs it also includes mouse and keyboard input and support for media application, presentation, even power functionalities. Web Share is another functionality of this application which is used to transfer file on HTTP [4] (browser). Where receiver can receive file on browser there no need to get client side Datawing application.

\section{LITERATURE REVIEW}

Through the literature review we came across certain papers which provided capability to the user to have remote access to laptop using android Smartphone. Various applications are available in the market that enables a user to have remote access to laptop. There are many problems faced by the presenter while navigating slides as he always needs to be dependent on another person for performing the job for him. Keeping all these drawbacks in mind we have decided to develop an application that will allow a user to access the PC remotely on android smartphones and sharing the files in the network between Wi-Fi compatible devices. a. Android based remote desktop client [1]: Android based remote desktop client is a remote control system which allows you to view and interact with one computer (known as server) to another computer or cellular phones (Android OS) anywhere ontheintranet. A prototype of the proposed system been implemented using Android and will be tested on a Android Virtual Device emulator.

All functions such mouse clicking, opening files, playing media can be perform on server computer. This system should able to communicate with the remote system and can find out whole description about the remote system and can have control on the peripheral devices connected to remote system from the mobile phones.

b. Remote Desktop Monitoring Using Android [2]: The key objective of the application is to remotely access and monitor different tasks running on the PC by connecting it to an Android operating system based mobile phone through a NETwork (internet). This application turns your mobile phone into a controller and viewer that can monitor the tasks running on PC and also view its desktop. Both the PC and mobile phone need to be connected to internet. . In this paper author can discuss the process of accessing and monitoring the computers with the help of android cellphones.

c. Android-Assisted Wireless Slide Cruising and File Transfer Application [3]: Android-Assisted Wireless Slide Cruising and File Transfer Application is designed to provide the users with the facility of transferring files across the net- work and remotely navigate a slide on PC as per the requirement.

This android application is capable of transferring commands to the server present on PC which will parse the request and perform corresponding action over Wi-Fi. So we propose to develop an application to access remote PC, transfer files at a faster speed and in a secure way as well as supportable for any platform. 


\section{III.PROPOSED SYSTEM}

A. Outline

1. Remote PC access[1]: The Wi-Fi compatible device will be used as remote control to control PPT, media player and various other applications on the computer.

2. File Share: In this application users select a file and click on send then the selected file will be transferred to a connected device. Also, this application is supportable to the WebShare[3].

3. WebShare[3]: In this user don't have a client side application of DataWing, in such case user will be sharing selected files using browser on client side device.

B. BlockDiagram of Proposed system

As shown in figure 1, the client and server side applications are connected with each other via WiFi network. They both are connected using socket programming. Client will access data from server and request for services and require information from server, respectively. Server will provide services to Client.

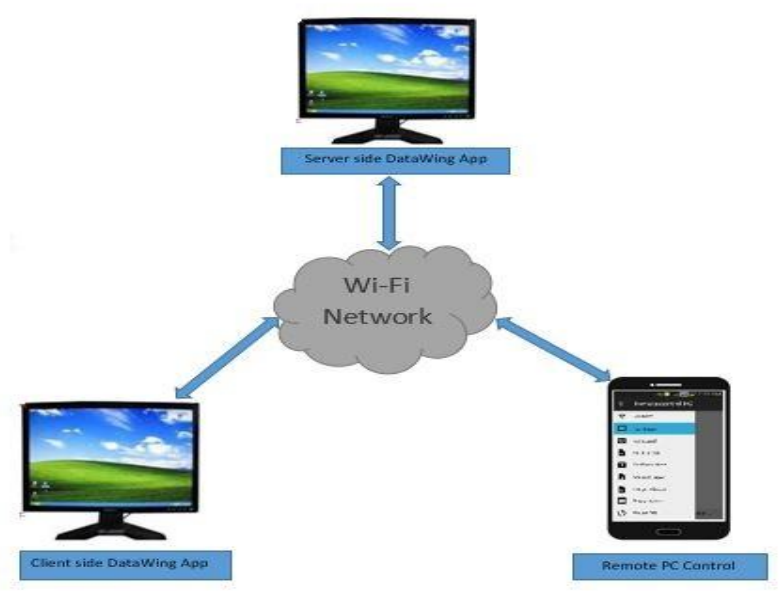

Fig. Block Diagram of DataWing File Sharing \& PC Control Application

\section{Different Modules in the application are as follows:}

1) WebShare:

The server side application will generate random IP address after connecting in network. That address will be put in the client side's web browser. Then client get directory which shared by server and from that client get files which he wants. From this way WebShare will be work for file sharing.

\section{2) Remote PC Control:}

In this module client side android application will be connect with server by WiFi network. Server generated IP will be put in the client side application for connecting with server. After that client will access PC remotely.

\section{3) FileShare:}

In this module both client and server side applications will connected with same network. Then client will be choose the files which sends to serer and just click on send to send it.

\section{Assumption}

The Users should have Wi-Fi Compatible device.

2) There should have users connected in same network. 3) Android 4.4 or above device for Remote PC functionality.

\section{IV.METHODOLOGY}

1.Socket Connections: A socket is one end point of a twoway communication link between two programs running on the network. A socket is bound to a port number so that the TCP layer can identify the application that data is destined to be sent to. An endpoint is a combination of an IP address and a port number. This socket connection will be used for connecting the client and server devices.

2. FTP(FileTransferProtocol): The File Transfer Protocol (FTP) is a standard network protocol used to transfer computer files between client and server on a computer network. FTP is built on a client-server model architecture and uses separate control and data connections between client and the server. So this protocol will be used in our application for client-server file sharing functionality.

3.HTTP (Hypertext Transfer Protocol): The Hypertext Transfer Protocol (HTTP)[4] is an application protocol for dis- tributed, collaborative, hypermedia information systems. HTTP is the foundation of data communication for the World Wide Web. Hypertext is structured text that uses logical links (hyperlinks) between nodes containing text. This protocol will be used in the WebShare file sharing functionality.

\section{IMPLEMENTATION}

A. File Sharing using FTP:

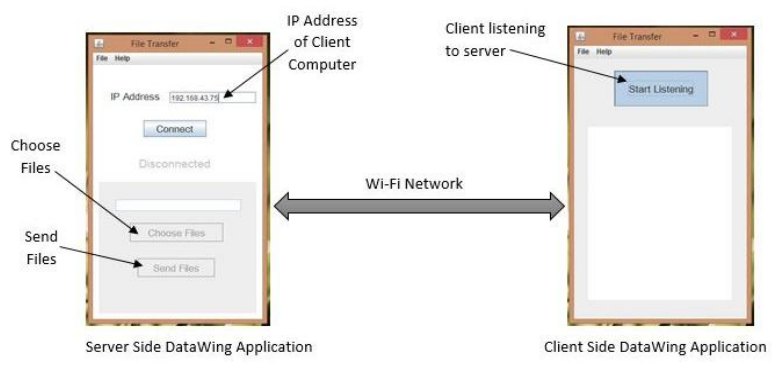

Fig. Working of File Sharing using FTP Functionality

The File Transfer using FTP is one functionality of DataWing Application. This Functionality working on windows platform PC. In this functionality client and server applications both are connected in same Wi-Fi network. Then using FTP Protocol they can transfer the files to each other. For that sender (i.e. server) can be put the IP address of receiver (i.e. client) in the first field .After 
connect to client using connect button and then choose file and send to client. When server connecting to client there at client side it can be listen to server's response.

B. Remote PC Control [1]:

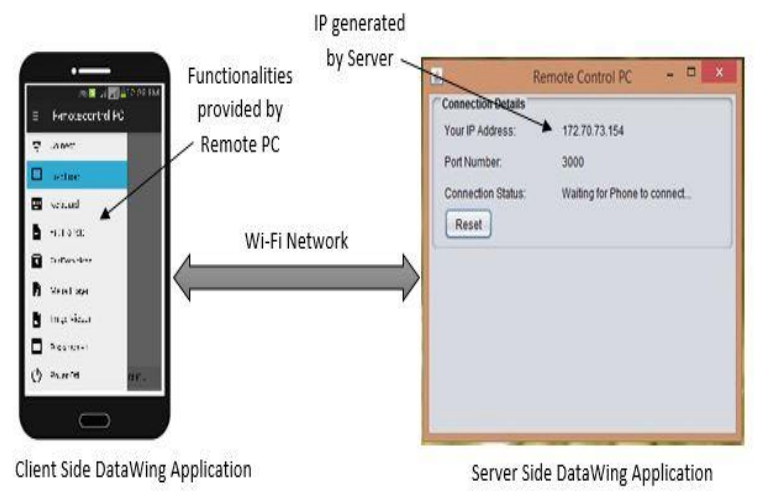

Fig. Working of Remote PC Control Functionality

Remote PC Control Functionality provides access of the computer to user through the android mobiles of user. In this the server side application of DataWing can run on PC. In the server application Randomly IP address can generate. This IP users want to put in the android Remote PC Control Application. By this IP address user making connection with PC to Android app. After connecting user can control PC action with the following functionalities:

1. Mouse touchpad:In this user can access the mouse pointer of PC. Also there left click and right click buttons are provided.

2. Keyboard:In this user can use application provided keyboard also the keyboard of his mobile to type any thing in computer's document or anywhere he requires.

3. Media Player:In this user can play the song from android mobile on the PC remotely.

4. Image Viewer:In this user can able to view images from its android mobile on the remote location PC.

5. Presentation:In this functionlity user ables to handle the PPT presentation through this android app from remote location.

6. Power Off: In this user ables to Power Off the PC which are connected with application.

Above all functionality can be provide using Wi-Fi network. For this application user no needs to root its android device.

\section{WebShare[3]:}

WebShare is a functionality which mainly introduce for file sharing in case if the client file sharing application is not present. In that case with the help of this functionality user can share files from web browser anywhere in the same network. There are on sender side (i.e. server side) application is present on PC. This application can generate random link. That link user can type in its web browser and go through this link.

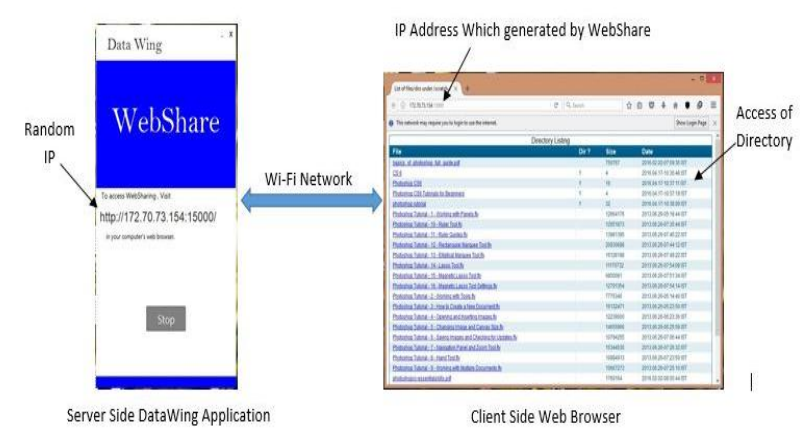

Fig. Working of WebShare Functionality

Then client can get all files from shared directory from sender and from this directory user will be download any file which they wants without internet connection. In this way without client side application user can share files using this functionality.

\section{CONCLUSION}

We can develop an application which will be able to transfer files from Wi-Fi device to another compatible WiFi device. In addition the application is including services like WebShare and Remote PC Control. The app will be offered faster speed than Bluetooth and better capability than NFC. It will also be able to secure itself from unauthenticated user transferring file.

\section{REFERENCES}

[1] Ajit Kotkar, Alok Nalawade, Siddhesh Gawas, Aniket Patwardhan, Snehal Mangale, "Android Based Remote Desktop Client", International Journal of Innovative Research in Computer and Communication Engineering ( Vol. 1), Issue 2, April 2013;

[2] Basu Shreejita, Kulkarni Pranita, Pawani Priyanka, "Remote Desktop Mon- itoring Using Android", International Journal of Innovative Research in Computer and Communication Engineering International Journal on Recent and Innovation Trends in Computing and Communication( Volume: 3),Issue: 3, March 2014;

[3] Prof. G. P. Mohole, Rohan Kulkarni, Amit Shinde, Vitthal Jadhav, Umesh Ahire, "Android Assisted Wireless Slide Cruising and File Transfer Application", International Journal of Advanced Research in Computer and Communication Engineering (Vol. 4), Issue 10, October 2015

[4] PeachPit Press, Kevin grant and Chris Haseman, "Beginning Android Programming DEVELOP AND DESIGN"

[5] The McGraw-Hill Companies, J.F. DiMarzio, "Android: A Programmers Guide"

[6] www.tutorialspoint.com/android/

[7] www.javatpoint.com/android-tutorial

[8] www.w3school.com 